This item was submitted to Loughborough's Research Repository by the author.

Items in Figshare are protected by copyright, with all rights reserved, unless otherwise indicated.

\title{
On nonlinear parametric amplification of Rayleigh waves
}

PLEASE CITE THE PUBLISHED VERSION

http://dx.doi.org/10.1016/0375-9601(93)90188-6

\section{PUBLISHER}

(c) Elsevier Science Publishers B.V.

\section{VERSION}

AM (Accepted Manuscript)

\section{LICENCE}

CC BY-NC-ND 4.0

\section{REPOSITORY RECORD}

Krylov, Victor V.. 2013. "On Nonlinear Parametric Amplification of Rayleigh Waves". figshare. https://hdl.handle.net/2134/12253. 
This item was submitted to Loughborough's Institutional Repository (https://dspace.lboro.ac.uk/) by the author and is made available under the following Creative Commons Licence conditions.

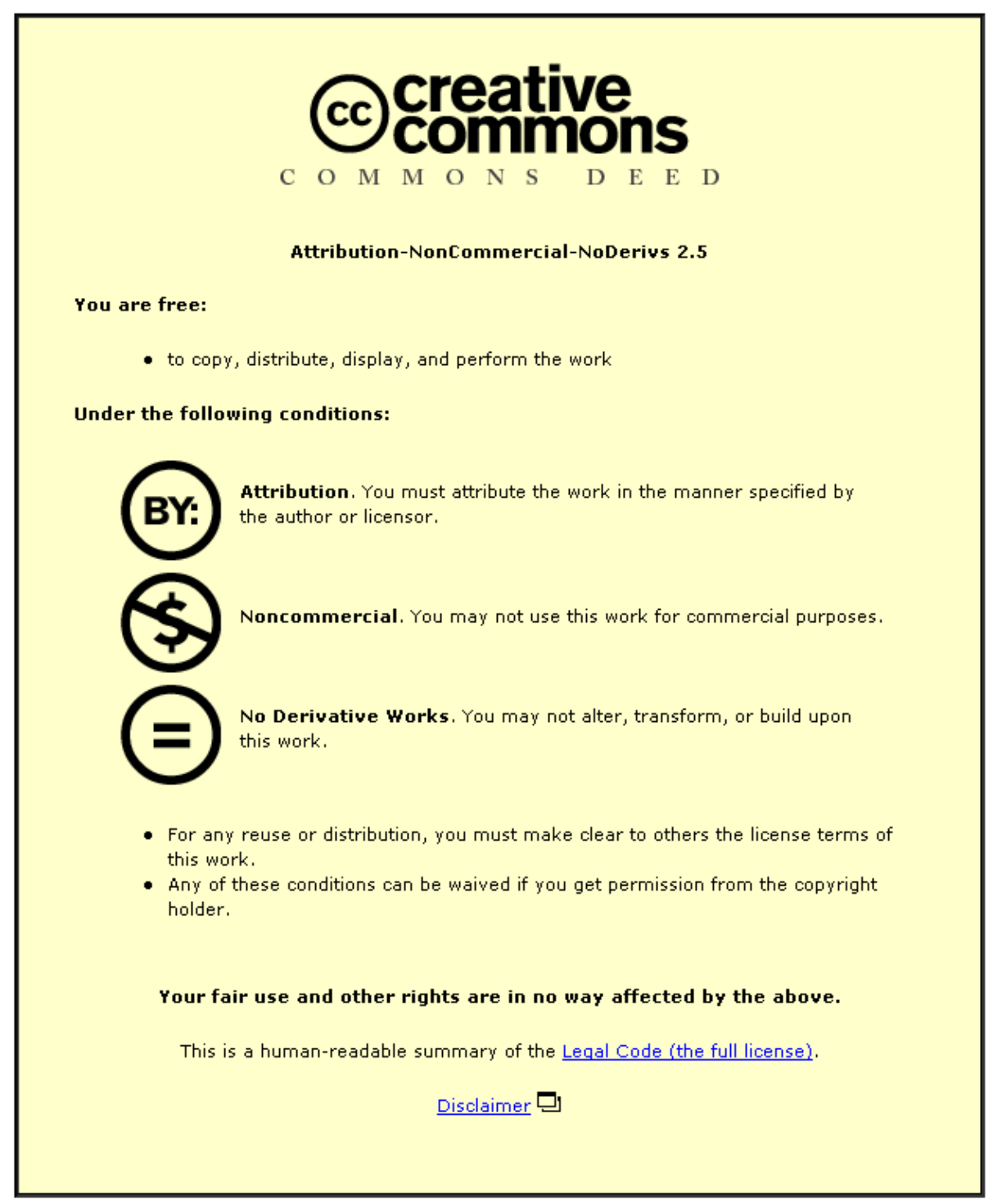

For the full text of this licence, please go to: http://creativecommons.org/licenses/by-nc-nd/2.5/ 
ON NONLINEAR PARAMETRIC AMPLIFICATION OF RAYLEIGH WAVES

V.V. Krylov *

Institut fuer Theoretische Physik, Universitaet Regensburg, Universitaetsstrasse. 31, D-8400 Regensburg, Germany

\section{ABSTRACT}

The phenomenon of parametric amplification of a weak Rayleigh surface acoustic wave of frequency $\omega_{0}$ by a strong pump Rayleigh wave of frequency $2 \omega_{0}$ is considered using the system of nonlinear evolution equations derived by D.F. Parker (Int. J. Engng Sci. 26 (1988) 59) and modified to take into account usual material dissipation and additional selective damping of certain wave harmonics. It is shown that introducing selective dissipation on the third and fourth harmonics together results in essentional increase of parametric amplification.

*) Permanent address: Department of Physics, Moscow M.V. Lomonosov State University, Moscow 117234, Russia 


\section{INTRODUCTION}

The phenomenon of nonlinear parametric amplification of a weak signal wave by a strong pump wave of a higher frequency is well known in optics and acoustics $[1,2]$. However, in contrast to optics, where because of strong dispersion some special cases may be chosen when waves of only two frequencies take part in the interaction (usually a signal wave of frequency $\omega_{0}$ and a pump wave of double frequency), the situation in acoustics is completely different. Usually the whole set of wave harmonics participates in this process because of very low dispersion. As a result, almost all energy of a strong pump wave is distributed between higher harmonics that causes the amplification of a signal wave to be rather small.

To improve this situation it has been suggested by several authors to introduce selective absorption on certain acoustic harmonics which restricts energy redistribution up to higher spectral components (see, e.g. [3]). A numerical analysis made in [3] for the case of bulk acoustic waves has shown that significant increase of parametric amplification may take place if to introduce appropriate selective attenuation on $3 \mathrm{rd}$ and/or 4 th harmonics.

It is known, however, that nonlinear effects are more easily pronounced in surface acoustic waves, in particular in Rayleigh waves in solids most part of their energy being localized in a relatively thin subsurface layer. 
This is why high wave amplitudes are more easily achieved (see reviews $[4,5])$. The aim of this work is to consider parametric amplification of Rayleigh surface acoustic waves and to investigate ways to make it more efficient.

Though many interesting results have been obtained for nonlinear propagation and waveform distortion of Rayleigh waves in the last decade [6-12], no attention has been paid to the parametric amplification of these waves which may be useful from a practical point of view. One can expect that since Rayleigh waves are dispersionfree and all their harmonics interact effectively with each other, the parametric amplification of a lower frequency signal wave will be rather low, like that for bulk acoustic waves. That is why some special methods should be used to increase amplification. In this question we will follow the ideas of the reference [3] investigating the effect of selective attenuation of certain wave harmonics on parametric amplification.

\section{BASIC EQUATIONS}

The problem of nonlinear Rayleigh wave propagation in elastic semispace $\mathrm{Z}<0$ can be described by the generalized equation of motion

$$
P_{0} u_{i}=T_{i j, j}
$$


with constitutive low that takes into account quadratic nonlinearity

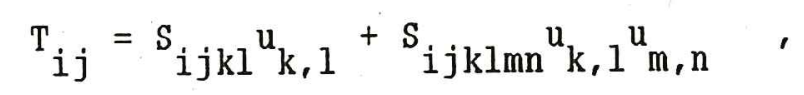

and by the stress-free boundary conditions on the free surface $\mathrm{Z}=0$ :

$$
\mathrm{T}_{i z}=0
$$

Here $u_{i}$ are the components of the displacement amplitudes, $T_{i j}$ are the components of the Piola - Kirchhoff stress tensor, $s_{i j k l}$ and $S_{i j k l m n}$ are components of linear and nonlinear $S$-tensors related to the linear $C_{i j k l}$ and nonlinear $C_{i j k l m n}$ components of elastic moduli [5], $\rho_{0}$ is a mass density in the state of equilibrium. In addition, $u_{i}$ and $T_{i j}$ must vanish as $\mathrm{Z}$ approaches - oo since the field of surface waves must vanish in the depth of the medium.

As is well known [5-12], a solution of (1)-(3) for the amplitude of vertical displacement $u_{z}$ at the surface of a solid $(z=0)$, which is the most relevant for practical purposes, can be represented as a Fourier series

$$
\left.u_{z}(\theta, x)=\sum_{n=1}^{\infty o} \mid D_{n}(X) \cos (n \theta)-E_{n}(X) \sin (n \theta)\right\}
$$


where $\theta=k_{0}\left(x_{1}-c_{R} t\right)$ is the phase variable, $x_{1}$ is a wave propagation distance, $\mathrm{k}_{0}=\omega_{0} / \mathrm{C}_{\mathrm{R}}$ is the wavenumber of the lowest wave harmonic, $\mathrm{C}_{\mathrm{R}}$ is the Rayleigh wave velocity, $D_{n}(X)$ and $E_{n}(X)$ are slowly varying amplitudes (the characteristic scale of their variation is determined by the nondimensional distance $\mathrm{X}=\mathrm{k}_{0} \varepsilon \mathrm{X}_{1}$, where $\varepsilon=\mathrm{k}_{0} \mathrm{C}_{0}, \mathrm{C}_{0}$ being a characteristic amplitude of the wave). Variation of $D_{n}$ and $E_{n}$ with the distance $X$ is described by the system of coupled evolution equations which determine spatial evolution of the amplitudes of harmonics and, hence, the form evolution of Rayleigh waves.

For the sake of simplicity, we restrict our consideration by the case of Rayleigh wave propagation in isotropic solid medium with only one nonzero Murnaghan nonlinear parameter (so called harmonic elastic material). For this case we will use the infinite system of coupled nonlinear evolution equations for isotropic elastic medium with the Poisson ratio $1 / 4$ derived by D.F. Parker $[10,11]$, but slightly modify them to include usual viscous dissipation, proportional to $\omega_{\mathrm{n}}^{2}$ (see also [12]), and additional selective dissipation of certain harmonics which may be caused, for example, by influence of periodic gratings on the surface.

Taking these into attention and using the notations of $[10,11]$, we may write the system of nonlinear evolution equations for the case under consideration in the form 


$$
\begin{aligned}
& \frac{d D_{n}}{d x}=-0.06715\left\{\sum_{m=1}^{n-1} \alpha_{m n} m(n-m)\left(D_{m} E_{n-m}+E_{m} D_{n-m}\right)+\right. \\
& \left.\sum_{m=1}^{\infty} \beta_{m n} m(n+m)\left(D_{m} E_{n+m}-E_{m} D_{n+m}\right)\right\}- \\
& P\left(u_{0}^{2} n^{2} D_{n}-Q(n) D_{n}\right. \\
& \frac{d E_{n}}{d x}=0.067151 \sum_{m=1}^{n-1} \alpha_{m n} m(n-m)\left(D_{m} D_{n-m}-E_{m} E_{n-m}\right)+ \\
& \left.\sum_{m=1}^{\infty o} \beta_{m n} m(n+m)\left(D_{m} D_{n+m}+E_{m} E_{n+m}\right)\right\}- \\
& P(1)_{0}^{2} n^{2} E_{n}-Q(n) E_{n}
\end{aligned}
$$

Here $\alpha_{m n}=1-\frac{2 n}{m+\Gamma(n-m)}, \quad \beta_{m n}=\frac{2 n}{m+n}-\frac{2 n}{n+\bar{I}}-\frac{2 m}{n+\Gamma m}$,

where $\Gamma=0.6339 ; \mathrm{P} \omega_{0}^{2}$ is a nondimensional constant depending on the properties of the medium and determining the viscous quadratic dissipation of wave energy; $Q(n)$ is a nondimensional constant determining additional selective dissipation imposing on the $\mathrm{n}$-th harmonic. In practice the infinite sums in (5) and (6) are replaced by finite ones with $N$ denoting the finite number of wave harmonics taken into account. The relations of the quantities $P 0_{0}^{2}$ and $Q(n)$ with widely used expressions for exponential attenuation $\sim \exp (-b \omega)_{n}^{2} x_{1}$ or $\sim \exp \left(-\alpha(n) x_{1}\right)$ are the 
following: $\quad P=b / k_{0} E$ and $Q(n)=\alpha(n) / k_{0} \Xi$.

\section{MAIN FEATURES OF RAYLEIGH WAVE PARAMETRIC AMPLIFICATION}

In this chapter we consider some specific features of parametric amplification of a weak signal Rayleigh wave of the main frequency $\omega_{0}$ by a strong pump wave of double frequency $2 \omega_{0}$ without introducing additional selective dissipation $(Q(n)=0)$. The initial waves at $X=0$ are considered as sine waves with amplitudes $E_{10}=0.01$ and $E_{20}=-1$ (as it is well known, the phase of a pump wave is paramount for obtaining amplification). The amplitudes $E_{30}, E_{40}, \ldots E_{N 0}$ as well as all amplitudes $\mathrm{D}_{10}, \mathrm{D}_{20}, \ldots \mathrm{D}_{\mathrm{NO}}$ of cosine components are taken to be zero. As it can be seen from the structure of the system $(5),(6)$ (see also $[10,11]$ ), in case of zero initial conditions for the amplitudes $D_{n}$ these amplitudes remain zero for all distancies $X$. Therefore, only equations (6) are really needed for the description of interacting waves.

It is interesting first to undertake a formal consideration of only two interacting harmonics $(N=2)$. In this case the system of nonlinear evolution equations (6) takes very simple form

$$
\frac{\mathrm{dE}}{\mathrm{dX}}=\mathrm{AE}_{1} \mathrm{E}_{2}-\mathrm{P} \mathrm{U}_{0}^{2} \mathrm{E}_{1}
$$




$$
\frac{\mathrm{dE}^{2}}{\mathrm{dX}}=\mathrm{BE}_{1}^{2}-4 \mathrm{Pu}_{0}^{2} \mathrm{E}_{2}
$$

where $\mathrm{A}=20.06715 \beta_{11}, \mathrm{~B}=-0.06715 \alpha_{12}$. This is similar to the case of nonlinear optics $[1,2]$ which possesses an exact analytical solution. The only difference is that the absolute value of the coefficient $B$ is two times smaller than that of the coefficient $A$, since $\beta_{11}=\alpha_{12}=$ -1.4481 . The reason of this can be explained by the nature of Rayleigh surface waves which are localized in depth of the order of their wavelenghts $\lambda_{n}=2 \pi C_{R} / \omega_{n}$, where $C_{R}$ is the Rayleigh wave velocity. Due to this fact, the energy transferred by a Rayleigh wave through an elastic semispace of unit width is proportional to the square of any of its amplitudes and to the first power of its frequency $\omega_{\mathrm{n}}$ (see, e.g.. [13]). In particular, if a Rayleigh wave is exdpanded into series of harmonics (4) with all $D_{n}(X)=0$, then the energy conservation low evidently has the form

$$
\frac{d}{d X}\left(\sum_{n=1}^{\infty} n E_{n}^{2}\right)=0
$$

It is easy to show that the relation $A=-2 B$ is exactly the sequence of the energy conservation low (9). Indeed, if we put $P=0$ in (7) and (8) (absence of dissipation) and then multiply equation (7) by $E_{1}$ and equation (8) - by $2 \mathrm{E}_{2}$, we will have after summation of the results the expression 


$$
\frac{d}{d x}\left(E_{1}^{2}+2 E_{2}^{2}\right)=0
$$

which coinsides with (9) if only two harmonics are considered. It follows from (10) that in the process of parametric amplification the initially weak signal wave may be more than $2^{1 / 2}$ times bigger in amplitude than the initial pump wave.

For small $\mathrm{X}$, when the amplitude of the pump wave $\mathrm{E}_{2}$ may be considered as a constant $\left(E_{2}=E_{20}\right)$, it follows from the equation (7) that

$$
\mathrm{E}_{1}=\mathrm{E}_{10} \exp \left\{\left(\mathrm{AE}_{20}-\mathrm{P}\left(\mathrm{U}_{0}^{2}\right) \mathrm{X}\right\},\right.
$$

i.e., in case of dissipation (nonzero value of $P$ ) the initial amplitude of the pump wave $\mathrm{E}_{20}$ should be higher than the gap level $\mathrm{E}_{20}^{(\mathrm{g})}=\mathrm{P}_{0}^{2} / \mathrm{A}$ in order the exponential growth of $E_{1}$ (i.e., the initial amplification) could be possible.

The behavior of $E_{1}$ and $E_{2}$ as functions of $X$ is shown on Fig.1 for the case of two interacting harmonics without and with material dissipation. In the first case $E_{1}$ grows until $X=29.02$ where $E_{1}$ takes the value $E_{1 \max }=1.414226$, slightly bigger than $2^{1 / 2} \quad$ (function $\quad E_{2}(X)$ goes through zero at this point). Then the classical picture of the second harmonic generation begins (relative to $\mathrm{E}_{2}$ ). The amplitude $\mathrm{E}_{2}$ tends asymptotically to a constant slightly exceeding one, whereas amplitude $E_{1}$ 
tends asymptotically to zero. In the presence of relatively small dissipation $\left(P \omega_{0}^{2}=0.01\right)$ the amplitude of the signal wave $E_{1}^{(D)}$ (index "D" is used to distinguish the dissipative case) reaches maximum at a longer distance $(X=47.5)$ and the value of this maximum is essentially lower $\left(E_{1 \max }^{(D)}=0.2997\right)$. Amplitude of the pump wave $E_{2}^{(D)}$ in this case goes through zero not in this point, but a little later. After that the reverse process of the second harmonic generation begins (for $\mathrm{E}_{2}^{(\mathrm{D})}$ ). Because of dissipation, the amplitude $\mathrm{E}_{2}^{(\mathrm{D})}$ has maximum at $\mathrm{X}=77.5$. Then it tends to zero together with $\mathrm{E}_{1}^{(\mathrm{D})}$.

The above discussed idealized picture of surface wave parametric amplification with accounting for only two interacting harmonics is usefull to evaluate the maximum achievable values of amplification both in elastic media with and without material dissipation. For the initial conditions and dissipation used the maximal coefficient of parametric amplification $\mathrm{K}_{\max }$ is equal to $\mathrm{E}_{1 \mathrm{max}} / \mathrm{E}_{10}=141$ in nondissipative case and $\mathrm{E}_{1 \mathrm{max}}^{(\mathrm{D})} / \mathrm{E}_{10}^{(\mathrm{D})}=$ 30 - in case of dissipation. However, in real situations of Rayleigh wave nonlinear propagation almost all harmonics usually take part in the interactions, and the above mentioned values of $\mathrm{K}_{\max }$ are never achieved.

The behavior of the amplitudes $E_{1}^{(D)}(X)$ and $E_{2}^{(D)}(X)$ with taking into account 20 interacting harmonics $(N=20)$ with the same parameter of dissipation $\left(\mathrm{P} \omega_{0}^{2}=0.01\right)$ has been investigated numerically via solution of the full system (5), (6) (with $Q(n)=0$ ) by modified Eulerian method. It has been shown in this case (see Fig.2 - solid curve "A") that maximal value of 
$E_{1}$ equal only to 0.03794 is achieved at $X=4.5$, i.e., at the distance a little bigger than the distance of a shock formation for Rayleigh waves $\mathrm{X}_{\mathrm{sh}}=3$ (see [6-11]). After this point the amplitude of a signal wave decreases since most of energy of the pump wave is spent for generation of higher harmonics. Thus, in this "natural" situation the parametric amplification is small $\left(\mathrm{K}_{\max }=3.794\right)$ and does not have any practical importance. The situation in this case is similar to that for bulk acoustic waves.

\section{INFLUENCE OF · SELECTIVE DAMPING}

It is obvious to expect that some improvement of the process of parametric amplification of Rayleigh waves may be obtained by introducing additional selective dissipation of certain harmonics as it was done for the case of bulk acoustic waves [3]. From practical point of view, realization of such selective dissipation for surface wave harmonics may be easier in comparizon with bulk acoustic waves. For example, one can make Rayleigh wave to propagate through periodic gratings on the surface [13] which may cause attenuation of corresponding harmonics due to their Bragg reflection or transversion into the bulk acoustic waves.

To investigate practically available conditions of increasing parametric amplification the numerical solution of the system (5), (6) with 
some $Q(n)$ different from zero has been carried out for several practically realistic combinations of selectively damped higher harmonics. In all cases under consideration the material (viscous) dissipation (with $\mathrm{P}_{0}^{2}=0.01$ ) was present. The initial conditions at $X=0$ were allways $E_{10}=0.01$ and $\mathrm{E}_{20}=-1$. Four different cases of selective dissipation have been considered: 1. Damping only third harmonic $(Q(3)=20) ; 2$. Damping only fourth harmonic $(Q(4)=20) ; \quad 3$. Damping third and fourth harmonics $(Q(3)=Q(4)=20) ; \quad$ 4. Damping third, fourth and fivth harmonics $(Q(3)=Q(4)=Q(5)=20)$.

The behavior of the amplitudes of the signal wave $E_{1}$ as functions of $\mathrm{X}$ for all these cases is shown on Fig.2 (solid lines). The behavior of corresponding amplitudes $\mathrm{E}_{2}$ is displayed on the same figure by dashed lines. As it can be seen from Fig.2, damping of only third harmonic (1) does not give any increase in parametric amplification if to compare this case with the case of absence of selective dissipation (curve A). This is in the contrast to the case of bulk acoustic waves [3] where damping third harmonic alone gives noticeable improvement. The result is even more discouraging if only fourth harmonic is damped (2). In this case the signal wave is almost not amplified at all.

However, introducing joint selective dissipation of third and fourth harmonics (3) gives rather good results, similarly to the corresponding situation for bulk acoustic waves [3]. Parameter $K_{\max }$ achieves in this case the value 18.60 at $X=51.3$, that is about 62 per cent of $K_{\max }$ for 
two interacting harmonics in dissipative medium (see previous chapter). Damping third, fourth and fivth harmonics together (4) gives not better results than damping third and fourth.

Thus, by making selective damping of third and fourth harmonics together a significant increase in parametric amplification of surface waves, interesting for applications, may be obtained. Practically such damping may be realized, for example, by creating on the surface a series of alternating gratings with periods providing Bragg reflection respectively for third and fourth harmonics of the amplified Rayleigh wave.

\section{ACKNOWLEDGEMENT}

The author is grateful to Dr. Andreas P. Mayer for stimulating discussions and for commenting on the manuscript. The author is also thankful to the University of Regensburg, Germany, for its Hospitality. 
REFERENCES

[1] M.B. Vinogradova, O.V. Rudenko and A.P. Sukhorukov, Theory of Waves (Nauka, Moscow, 1979) - in Russian.

[2] N. Bloembergen, Nonlinear Optics (Benjamin, Inc., New York, 1965).

[3] V.G. Andreev, O.A. Vasilieva, E.A. Lapshin and O.V. Rudenko, Akust. Zh., $31(1985) 12$.

[4] G.I. Stegeman and F. Nizzoli, in "Surface Excitations", V.M. Agranovich and R. Loudon, eds. (Elsevier, Amsterdam, 1984), p. 195.

[5] A.A. Maradudin and A.P. Mayer, in "Nonlinear Waves in Solid State Physics", A.D. Boardman, T. Twardowski and M. Bertolotti, eds. (Plenum, New York, 1990), p. 113.

[6] N. Kalyanasundaram, Int. J. Engng. Sci. 19 (1981) 279.

[7] R.W. Lardner, Int. J. Engng. Sci. 21 (1983) 1331.

[8] M. Planat, J. Appl. Phys. 57 (1985) 4911.

[9] D.F. Parker and F.M. Talbot, J. Elast. 15 (1985) 389.

[10] D.F. Parker, Int. J. Engng. Sci. 26 (1988) 59.

[11] D.F. Parker, Physics of the Earth and Planetary Interiors 50 (1988) 16.

[12] D.F. Parker and A.P. Mayer, in "Nonlinear Waves and Dissipative Effects", D. Fusco and A. Jeffrey, eds. (Longman, Harlow, 1991), p.42.

[13] S.V. Biryukov, Yu.V. Gulyaev, V.V. Krylov and V.P. Plessky, Surface Acoustic Waves in Inhomogeneous Media (Nauka, Moscow,1991).-in Russian 
FIGURE CAPTIONS

Fig.1 Amplitudes of signal (solid lines) and pump (dashed lines) surface acoustic waves as functions of $\mathrm{X}$ for accounting only two lowest wave harmonics. Index "D" denotes corresponding curves in the presence of material dissipation

Fig.2 Amplitudes of signal (solid lines) and pump (dashed lines) surface acoustic waves in dissipative medium as functions of $\mathrm{X}$ for the cases: A - Absence of additional selective damping; 1 .- Damping only third harmonic; 2. - Damping only fourth harmonic; 3. - Damping third and fourth harmonic; 4. - Damping third, fourth and fivth harmonic. Interaction of 20 harmonics have been taken into account. 


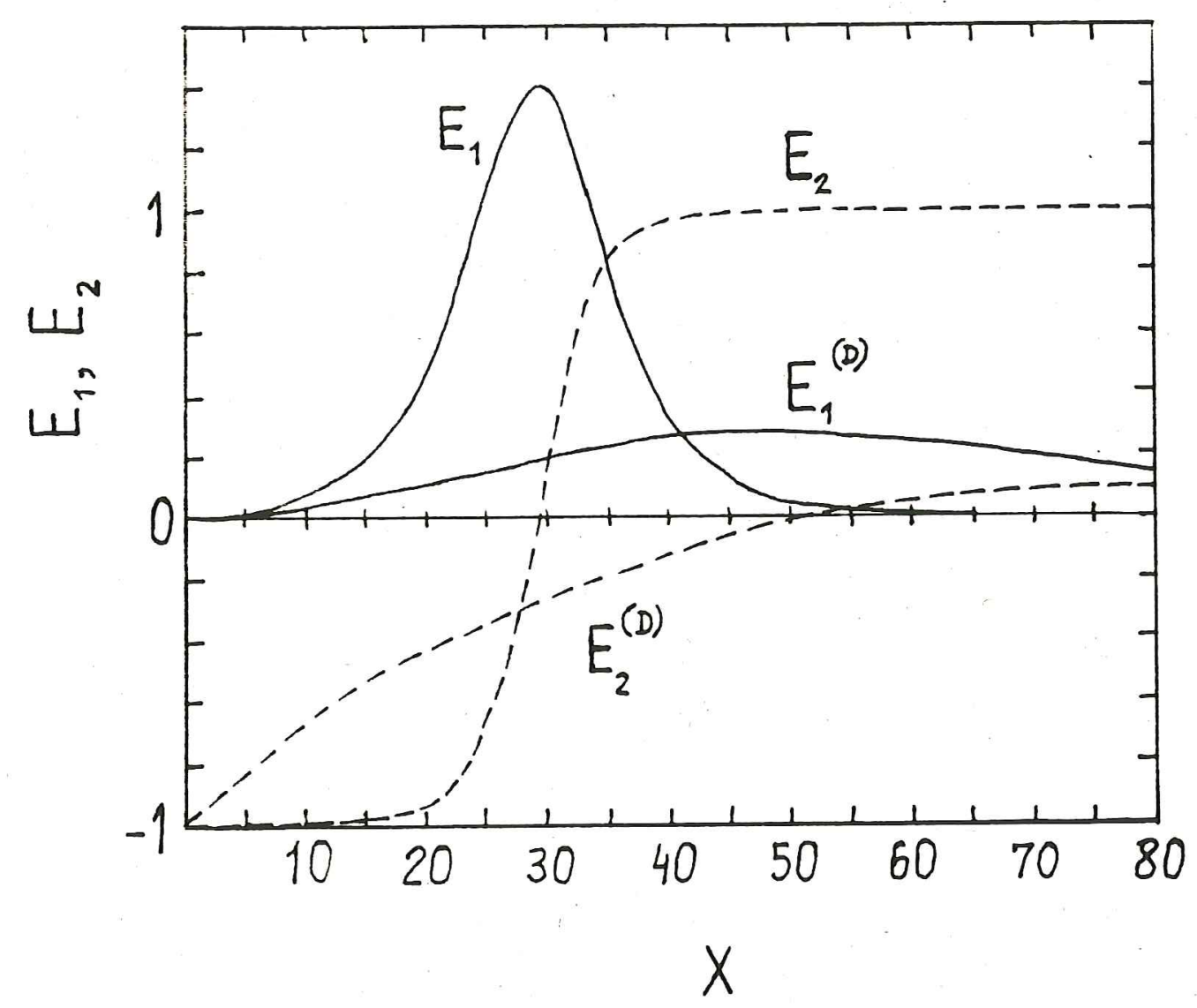

Fig. 1 


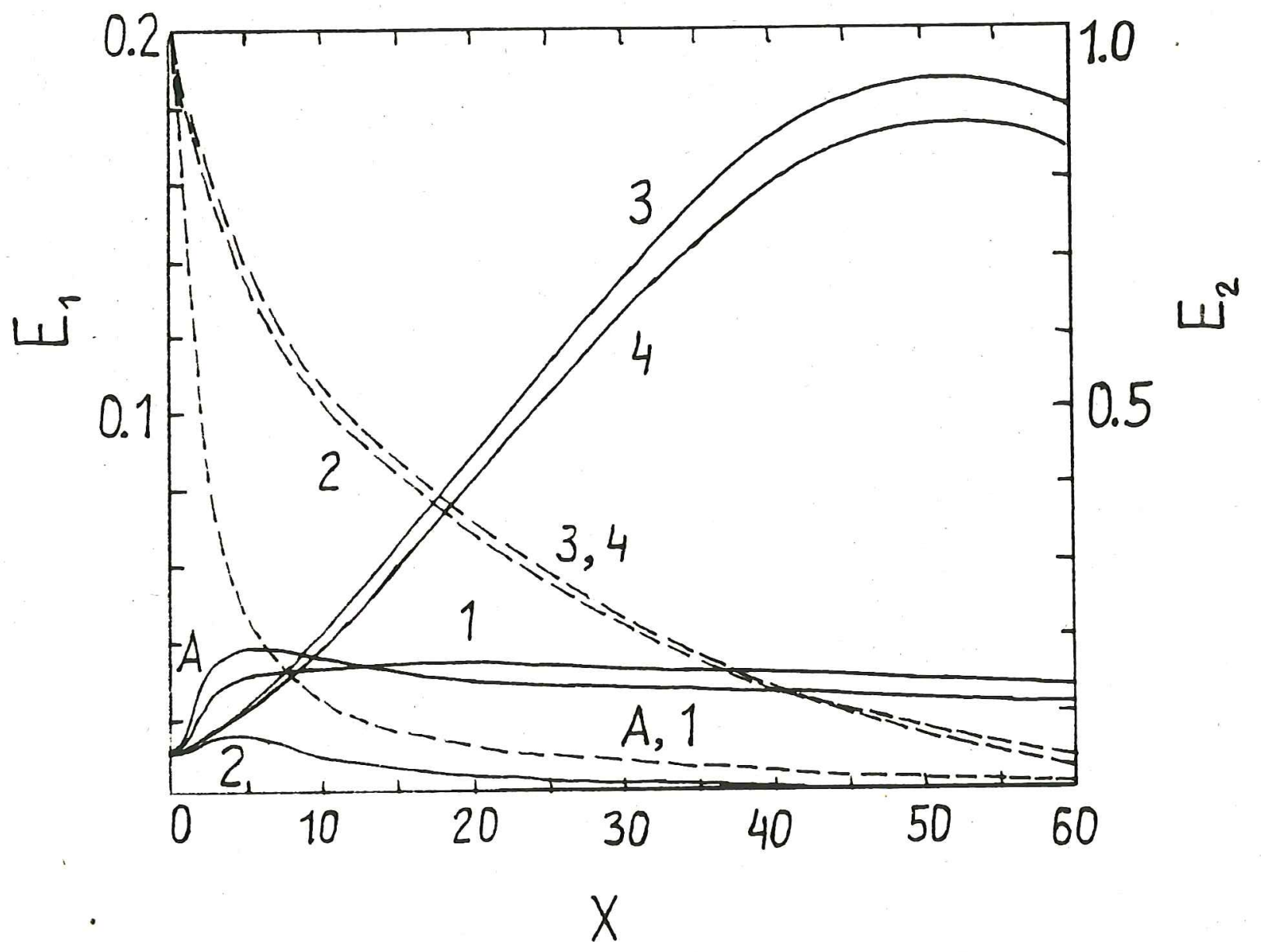

Fig. 2 